\title{
Descarga Interna e Tensão de Retorno em Capacitores
}

Internal discharge and return voltage in capacitors

\author{
Renê Robert \\ Departamento de Engenharia Elétrica da UFPR \\ C.P. 19047 Curitiba, PR, 81531-990
}

Recebido em 7 de Dezembro 2000. Manuscrito revisado em 23 de Agosto 2001. Aceito em 06 de Setembro 2001.

\begin{abstract}
Os fenômenos de descarga interna e tensão de retorno em capacitores são calculados no caso onde a resposta do dielétrico é da forma exponencial no tempo. Com esta hipótese a matemática envolvida é simples e as características gerais dos fenômenos de hereditariedade podem ser seguidas.
\end{abstract}

The phenomena of internal discharge and return voltages of capacitors are studied for the case where the dielectric response in time domain is of exponential type. The mathematics involved becomes sufficiently simple and the general feature of the heredity phenomena may be followed.

\section{Introdução}

No estudo de capacitores não se mencionam alguns fenômenos que ocorrem normalmente em dielétricos sólidos e líquidos. Considere o circuito da Fig. 1. Carregamos o capacitor $\mathrm{C}$ colocando a chave $\mathrm{S}$ na posição 1 durante um tempo suficientemente longo $\xi$ e a seguir colocando a chave $\mathrm{S}$ na posição 0 , observa-se experimentalmente no eletrômetro $\mathrm{E}$ que a diferença de potencial nas placas do capacitor diminui muito lentamente. Este fenômeno chama-se descarga interna. Um segundo experimento consiste em se colocar a chave $\mathrm{S}$ na posição 1 durante um intervalo suficientemente longo $\xi$ e a seguir a chave $\mathrm{S}$ é colocada na posição 2, isto é, curto-circuitando o capacitor durante um intervalo de tempo $\eta$ de alguns segundos, passando então a chave $\mathrm{S}$ para a 0 . A observação do eletrômetro $\mathrm{E}$ mostra que a diferença de potencial inicialmente nula aumenta até atingir um valor máximo, que é uma fração da tensão de polarização, para a seguir tender a zero muito lentamente. Este fenômeno é conhecido como tensão de retorno pode ser danoso para pessoas desavisadas que trabalham em instalações de alta tensão como por exemplo bancos de capacitores. Nas Figs. 2 e 3 mostra-se esquematicamente os fenômenos acima descritos. Estes fatos já eram conhecidos desde o século XIX, quando Hopkinson (1877)[1] os estudou por sugestão de J.C. Maxwell. Na literatura técnica estes e outros fenômenos similares receberam nomes diversos como after-effect [2], proposto por L. Boltzmann na teoria elástica, ou efeitos de hereditariedade [3] proposto por V. Volterra. Do ponto de vista da eletricidade estes fenômenos são importantes pois estão relacionados com perdas dielétricas [4] e mostram que o capacitor tem um comportamento razoavelmente mais complexo que aquele descrito nos livros de texto.

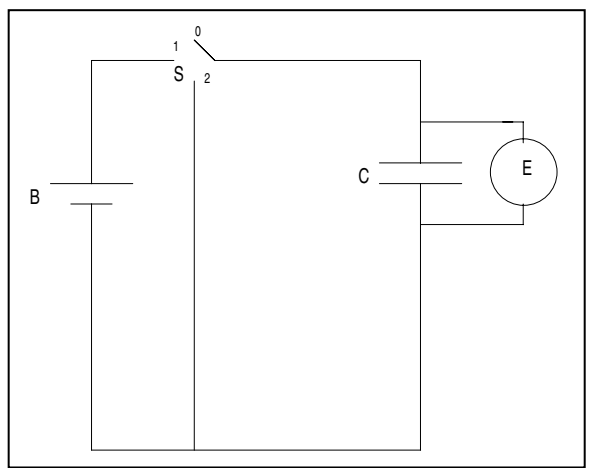

Figura 1. Circuito elétrico usado para as medidas da descarga interna e da tensão de retorno do capacitor C.

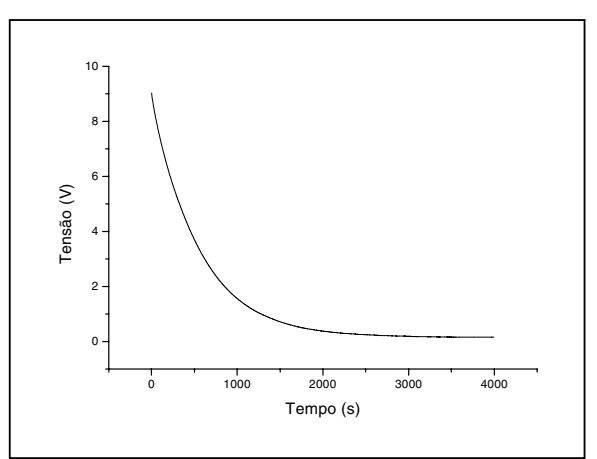

Figura 2. Descarga interna de um capacitor de mica de 1nF tempo de polarização $\xi=19 \mathrm{~h}, U_{0}=9,53 \mathrm{~V}$. 


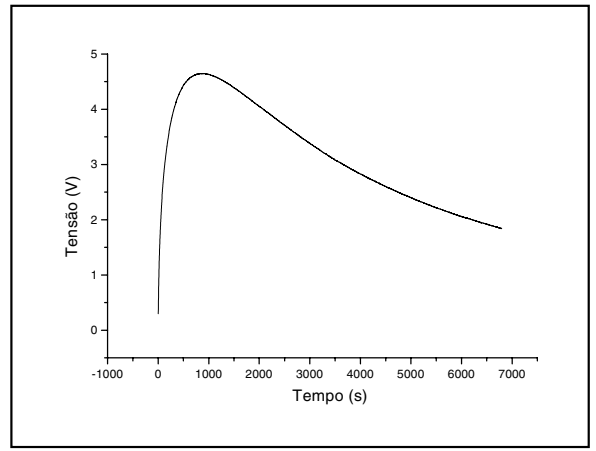

Figura 3. Tensão de retorno de um capacitor de mica com $\mathrm{C}=0,68$ tempo de polarizaçãp $\xi=30 \mathrm{~min}$, tempo de curtocircuito $\eta=5 \mathrm{~s}$ e $U_{0}=102 \mathrm{~V}$.

Em 1937 B. Gross [5], a partir de considerações de teoria de circuitos, desenvolveu um formalismo que permite calcular a corrente $I(t)$ que atravessa o dielétrico de um capacitor de placas planas e paralelas, depois de solicitado por uma tensão continuamente variável a partir do instante $t=0$, a qual é dada pela equação

$I(t)=\frac{U(t)}{R}+C_{0} \frac{d U(t)}{d t}+I_{0}(t)+\int_{0}^{t} \frac{d U(\tau)}{d \tau} \varphi(t-\tau) d \tau$,

em que $R$ é a resistência elétrica do dielétrico, $C_{0}$ a capacitância geométrica, $\varphi(t)$ uma função que caracteriza o dielétrico chamada função relaxação dielétrica com as condições de ser nula no infinito e para valores negativos do tempo, $I_{0}(t)=\int_{-\infty}^{0} \frac{d U(\tau)}{d \tau} \varphi(t-\tau) d \tau$ é a corrente gerada por todas as variaçồes de tensão anteriores ao instante inicial, que traduz o fenômeno de hereditariedade e por esta razão chamada corrente hereditária. Os dois problemas anteriormente mencionados, da descarga interna e da tensão de retorno podem se resolvidos pela Eq. (1), bastando para tal fazer a corrente externa $I(t)=0$. Desta forma obtémse uma equação integro-diferencial para a função $U(t)$. Uma solução para esta equação integro-diferencial foi obtida por B. Gross [5] quando a função relaxação dielétrica é da forma $\varphi(t)=\varphi_{0} \exp (-\mu t)$, onde $\varphi_{0}$ e $\mu$ são constantes que caracterizam o dielétrico. Embora os dielétricos reais não sejam regidos por uma função relaxação dielétrica deste tipo [6] os resultados qualitativos são bons e a matemática envolvida é razoavelmente simples.

Nosso objetivo é múltiplo: em primeiro lugar mostrar como a teoria de dielétricos pode ser tratada do ponto de vista fenomenológico de maneira mais precisa que aquela encontrada normalmente nos livros de texto; segundo mostrar como resolver uma equação integrodiferencial usando como função relaxação dielétrica uma função exponencial no tempo (Debye) e comparar os resultados obtidos com aqueles experimentais; terceiro, lembrar um pouco da história da física no Brasil enfatizando que este formalismo foi desenvolvido inicialmente pelo prof. B. Gross; quarto lembrar que este é um problema clássico ainda não resolvido completamente e que apresenta grande interesse técnico na área de envelhecimento de materiais dielétricos em cabos e transformadores. Em suma procurou-se popularizar os efeitos de um dielétrico real, sejam eles sólidos, amorfos ou cristalinos ou líquidos.

\section{Desenvolvimento teórico}

A partir do vetor densidade de corrente elétrica total (densidade de corrente ôhmica mais densidade de corrente de deslocamento), vamos deduzir a Eq. (1) a partir de considerações teóricas fenomenológicas. Usase aqui a mesma conceituação utilizada for R. E. Tilley [7] que consiste em supor que a polarização total do dielétrico num ponto seja composta de duas partes: $\vec{P}_{i}(t)=\epsilon_{0} \chi_{i} \vec{E}(t)$, a qual responde ao campo elétrico $\vec{E}(t)$ de maneira quase instantânea; $\vec{P}_{s}(t)=$ $\epsilon_{o} \int_{0}^{\infty} \chi_{s}(\lambda) \vec{E}(t-\lambda) d \lambda$; polarização lenta, que obedece aos princípios de causalidade e superposição e depende de todos os campos elétricos previamente existentes ao instante $t$ (vide apêndice). Sendo $\vec{P}(t)=\vec{P}_{i}(t)+\vec{P}_{s}(t)$ e definindo a suscetibilidade generalizada no ponto em questão pela equação $\chi(t)=\chi_{i} \delta(t)+\chi_{s}(t)$, pode-se escrever o vetor polarização total pela equação

$$
\vec{P}(t)=\epsilon_{0} \int_{-\infty}^{t} \chi(t-\tau) \vec{E}(\tau) d \tau .
$$

A explicação para o desdobramento da polarização elétrica em duas parcelas está relacionada aos diversos mecanismos propostos para o fenômeno de polarização em um dielétrico, dos quais os mais importantes são: polarização eletrônica, polarização iônica; polarização dipolar; polarização interfacial nos eletrodos e nas vizinhanças de inomogeneidades; injeção de cargas induzindo efeitos de carga espacial; tunelamento de portadores de carga para armadilhas e salto de portadores de carga de um estado localizado para outro. Alguns destes processos são extremamente rápidos como ocorre na polarização eletrônica e na polarização iônica, traduzidos através de $\chi_{i}$, outros são mais lentos e são os responsáveis pelo efeitos de hereditariedade, traduzidos aqui por $\chi_{s}(t)$.

Supondo que o dielétrico tem condutividade elétrica $\sigma$ e obedece a lei de $\mathrm{Ohm}$ pode-se calcular o vetor densidade de corrente elétrica total que é dado pela equação

$$
\vec{J}(t)=\sigma \vec{E}(t)+\epsilon_{0}\left(1+\chi_{i}\right) \frac{d \vec{E}(t)}{d t}+\epsilon_{0} \int_{-\infty}^{t} \chi_{s}(t-\tau) \frac{d \vec{E}(\tau)}{d \tau} d \tau
$$


A Eq. (3) pode ser particularizada para o caso de um capacitor com dielétrico homogêneo, de placas planas e paralelas de área $A$ e espessura $\ell$ com a condição $A>>\ell^{2}$ obtendo-se

$$
I(t)=\frac{U(t)}{R}+\epsilon_{\infty} C_{0} \frac{d U(t)}{d t}+C_{0} \int_{0}^{t} \chi_{s}(t-\tau) \frac{d U(\tau)}{d \tau} d \tau+I_{0}(t)
$$

em que $\epsilon_{0}$ é a permissividade do vácuo, $\epsilon_{\infty}=1+\chi_{i}$ é a permissividade relativa a alta freqüência, $C_{0}=\frac{\epsilon_{0} A}{\ell}$ a capacitância geométrica e $I_{0}(t)=C_{0} \int_{-\infty}^{0} \chi_{s}(t-$ $\tau) \frac{d U(\tau)}{d \tau} d \tau$ a corrente hereditária gerada por todas as variações de tensão anteriores ao instante inicial. A Eq. (4) é formalmente semelhante à Eq. (1) proposta por B. Gross.

No caso particular de circuito externo aberto, $I(t)=$ 0 , e chamando $\psi(t)=\frac{d U(t)}{d t}$, a Eq. (4) se transforma numa equação integral de Volterra de 2a. espécie [8] e pode ser escrita como

$$
\psi(t)+\int_{0}^{t}\left[\lambda+\frac{\varphi(t-\tau)}{C_{\infty}}\right] \psi(\tau) d \tau=f(t)
$$

em que $C_{\infty}=\epsilon_{\infty} C_{0}, \lambda=\frac{1}{R C_{\infty}}, \varphi(t)=$ $C_{0} \chi_{s}(t)$, chamada função relaxação dielétrica e $f(t)=$ $-\left[\frac{I_{0}(t)}{C_{\infty}}+\lambda(U)(0)\right]$.

Para resolver a Eq. (5) vamos inicialmente resolver a equação de Volterra fazendo no segundo membro a substituição de $f(t)$ pela função delta de Dirac $\delta(t)$. Com esta transformação obtém-se

$$
G(t)+\int_{0}^{t}\left[\lambda+\frac{\varphi(t-\tau)}{C_{\infty}}\right] G(\tau) d \tau=\delta(t) .
$$

É fácil mostrar que a solução da Eq. (5) fica determinada pela equação

$$
\psi(t)=\int_{0}^{t} G(t-\tau) f(\tau) d \tau
$$

Conseqüentemente a solução da Eq. (4) fica na forma

$$
U(t)-U(0)=\int_{0}^{t} d \tau \int_{0}^{\tau} G(t-\lambda) f(\lambda) d \lambda
$$

Usando como função de relaxação dielétrica a mesma expressão exponencial usada por B. Gross[5] e utilizando o método das transformadas de Laplace em ambos os membros da Eq. (6) obtém-se

$$
G(t)=\delta(t)+\alpha \frac{\alpha+\mu}{\alpha-\beta} \exp (\alpha t)-\beta \frac{\beta+\mu}{\alpha-\beta} \exp (\beta t)
$$

em que

$$
\begin{aligned}
& \alpha=-\frac{1}{2}(\lambda+\mu+\kappa)+\frac{1}{2} \sqrt{(\lambda+\mu+\kappa)^{2}-4 \lambda \mu}, \\
& \beta=-\frac{1}{2}(\lambda+\mu+\kappa)-\frac{1}{2} \sqrt{(\lambda+\mu+\kappa)^{2}-4 \lambda \mu},
\end{aligned}
$$

e

$$
\kappa=\frac{\varphi_{0}}{C_{\infty}}
$$

Sendo $\xi$ e $U_{0}$, respectivamente o tempo e a tensão de polarização, a Eq. (8) pode ser resolvida com auxilio da identidade

$$
\int_{0}^{x} d t \int_{0}^{t} \varphi(t-\tau) \frac{d \psi(\tau)}{d \tau} d \tau=-\psi(0) \int_{0}^{x} \varphi(t) d t+\int_{0}^{x} \varphi(x-t) \psi(t) d t
$$

obtendo-se para a tensão de descarga interna a equação

$$
U(t)=\frac{U_{0} \kappa}{\alpha-\beta}\left\{\exp (\beta t)\left[\exp (-\mu \xi)+\frac{\lambda(\beta+\mu)}{\beta \kappa}\right]-\exp (\alpha t)\left[\exp (-\mu \xi)+\frac{\lambda(\alpha+\mu)}{\alpha \kappa}\right]\right\}
$$

em que fizemos $f(t)=-U_{0}[\lambda+\kappa \exp (-\mu(t+\xi))] \mathrm{e}$ $U(0)=U_{0}$.

De maneira análoga, obtém-se para a tensão de re- torno com tensão de polarização $U_{0}$, tempo de polarização $\xi$ e tempo de curto-circuito $\eta$ a seguinte equação 
$U(t)=\frac{U_{0} \kappa \exp (-\mu \eta)}{\alpha-\beta}[1-\exp (-\mu \xi)][\exp (\alpha t)-\exp (\beta t)]$,

em que fizemos $f(t)=U_{0} \kappa[\exp (-\mu(t+\eta))-\exp (-\mu(t+$ $\xi+\eta))]$ e $U(0)=0$.

As Eqs. (4), (10) e (11) apresentam algumas conseqüências importantes do ponto de vista experimental:

- O máximo da tensão de retorno na Eq. (11), ocorre no instante $t^{*}=\frac{\ln (\alpha / \beta)}{\alpha-\beta}$ o qual independe do tempo de polarização e do tempo de curto-circuito;

- As Eqs. (10) e (11) não são completamente independentes. É possível deduzir uma a partir de outra. Considere a Eq. (10) aplicada a dois tempos de polarização $\xi_{1}$ e $\xi_{2}$. Por subtração obtém-se a Eq. (11) para um tempo de polarização $\xi=\xi_{1}-\xi_{2}$ e tempo de curto circuito $\eta=\xi_{2}$. Esta propriedade foi mostrada teórica e experimentalmente por B. Gross[9];

- Polarizando o dielétrico durante um intervalo de tempo $\xi$ a Eq. (4) permite calcular a capacitância

$$
C(\xi)=C_{\infty}+\int_{0}^{\infty} \varphi(t) d t-\int_{0}^{\infty} \varphi(t+\xi) d t,
$$

a qual depende do tempo de polarização. Quando $\xi \rightarrow \infty$ obtém-se

$$
C(\infty)=C_{\infty}+\int_{0}^{\infty} \varphi(t) d t
$$

em que a parcela $\int_{0}^{\infty} \varphi(t) d t=\frac{\varphi_{0}}{\mu}$ é chamada capacitância anômala;

- Um caso interessante ocorre quando o dielétrico é um isolante perfeito, isto é, $R \rightarrow \infty$. Nestas condições as Eqs. (10) e (11) se transformam respectivamente em

$$
U(t)=\frac{U_{0}}{\mu+\kappa}\{\kappa \exp (-\mu \xi)[\exp (-(\mu+\kappa) t)-1]+\mu+\kappa\},
$$

$$
U(t)=\frac{U_{0} \kappa \exp (-\mu \eta)}{\mu+\kappa}(1-\exp (-(\mu+\kappa) t)) .
$$

Quando a carga do capacitor for completa, $\xi \rightarrow \infty$, verificamos que o capacitor não sofre descarga interna $\mathrm{e}$ a tensão de retorno, após um intervalo de tempo muito grande atinge o valor $\frac{U_{0} \kappa}{\mu+\kappa} \exp (-\mu \eta)$. Este resultado nos diz que o termo da resistência na corrente total promove a descarga interna e se opõe à tensão de retorno levando esta a zero.

\section{Resultados numéricos}

A Fig. 4 mostra o ajuste da Eq. (11) com os dados experimentais obtidos para a tensão de retorno em um filme de polietileno com eletrodos de alumínio, tempo de polarização $\xi=15 \mathrm{~h}, 50 \mathrm{~min}$, tempo de curto-circuito $\eta=5$ s e tensão $U_{0}=158,4 \mathrm{~V}$. Este ajuste permite obter os parâmetros que aparecem na Eq. (11). A Fig. 5 mostra o ajuste da Eq. (10) com os dados experimentais da curva de descarga interna para a mica moscovita com eletrodos de prata. O tempo de polarização para este caso é $\xi=20 \mathrm{~h}$ e a tensão $U_{0}=9,55 \mathrm{~V}$. Da mesma forma que no caso anterior o ajuste permite obter os parâmetros da Eq. (10). Embora a teoria aqui desenvolvida não seja quantitativamente correta ela apresenta um bom resultado do ponto de vista qualitativo. A tabela I mostra os resultados do ajuste com os dados experimentais, em unidades do Sistema Internacional.

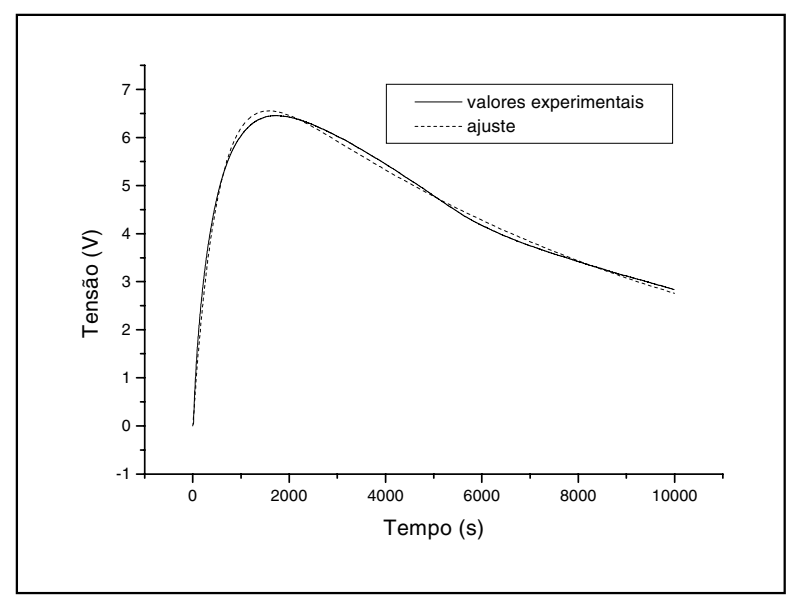

Figura 4. Tensão de retorno em um filme de polietileno com $\xi=15 \mathrm{~h} 50 \mathrm{~min} \eta=5$ s e $U_{0}=158,4 \mathrm{~V}$. A capacitância da amostra é de $332 \mathrm{pF}$.

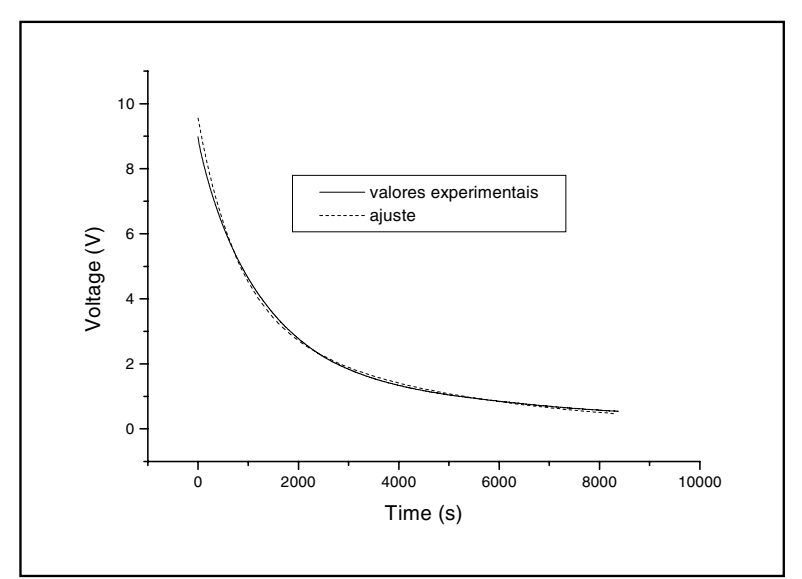

Figura 5. Descarga interna em uma amostra de mica moscovita com eletrodo de prata $\xi=20 \mathrm{~h}, U_{0}=9,55 \mathrm{~V}$. A capacitância da amostra é $0,824 \mathrm{nF}$. 


\begin{tabular}{|c|c|c|c|c|c|}
\hline Dielétrico & $\mu\left(\mathrm{s}^{-1}\right)$ & $\lambda\left(\mathrm{s}^{-1}\right)$ & $\kappa\left(\mathrm{s}^{-1}\right)$ & $R(\Omega)$ & $\varphi_{0}(\mathrm{~S})$ \\
\hline Polietileno & 0,0001158 & 0,0018241 & 0,0000948 & $1,5910^{12}$ & $3,310^{-14}$ \\
\hline Mica & 0,0003637 & 0,0008782 & 0,0002893 & $3,7310^{12}$ & $8,810^{-14}$ \\
\hline
\end{tabular}

A Fig. 6 mostra a tensão de retorno em um filme de polietileno para dois tempos de polarização diferentes $\xi=\infty$ e $\xi=1800$ s com tempo de curto-circuito $\eta=5 \mathrm{~s}$.

A Fig. 7 mostra a tensão de descarga interna para a amostra de mica moscovita para dois tempos de polarização diferentes $\xi_{1}$ e $\xi_{2}=5 \mathrm{~s}$. Mostra-se que pela diferença das duas curvas de descarga interna obtêm-se a tensão de retorno para $\xi=1795$ s e $\eta=5$ s.

A Fig. 8 mostra as curvas de tensão de retorno e descarga interna em um dielétrico perfeito $(R \rightarrow \infty)$ para dois tempos de polarização finitos e distintos.

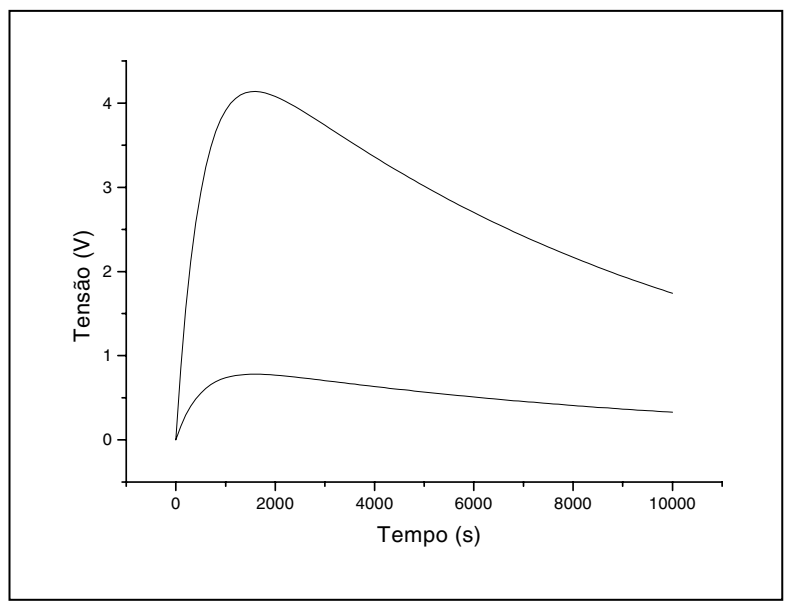

Figura 6. Tensão de retorno calculada para dois tempos de polarização, $\xi=\infty$ e $\xi=1800$ s em uma amostra de polietileno com tempo de curto-circuito $\eta=5 \mathrm{~s}$. Tensão $U_{0}=100 \mathrm{~V}$.

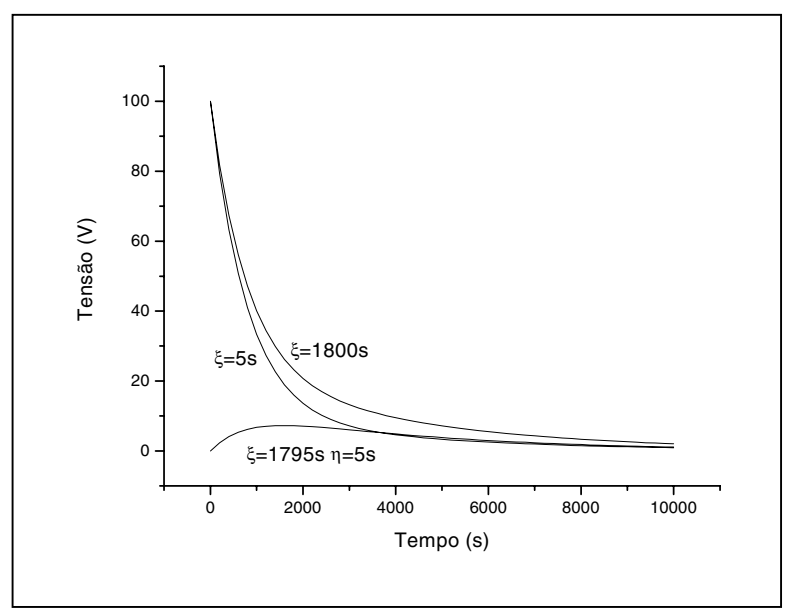

Figura 7. Descarga interna calculada para dois diferentes tempos de polarização, $\xi_{1}=1800$ e $\xi_{2}=5$ s em uma amostra de mica moscovita com tensão $U_{0}=100 \mathrm{~V}$. A tensão de retorno foi obtida pela diferença entre as duas curvas de descarga interna onde $\xi=1795 \mathrm{~s}$ e $\eta=5 \mathrm{~s}$.

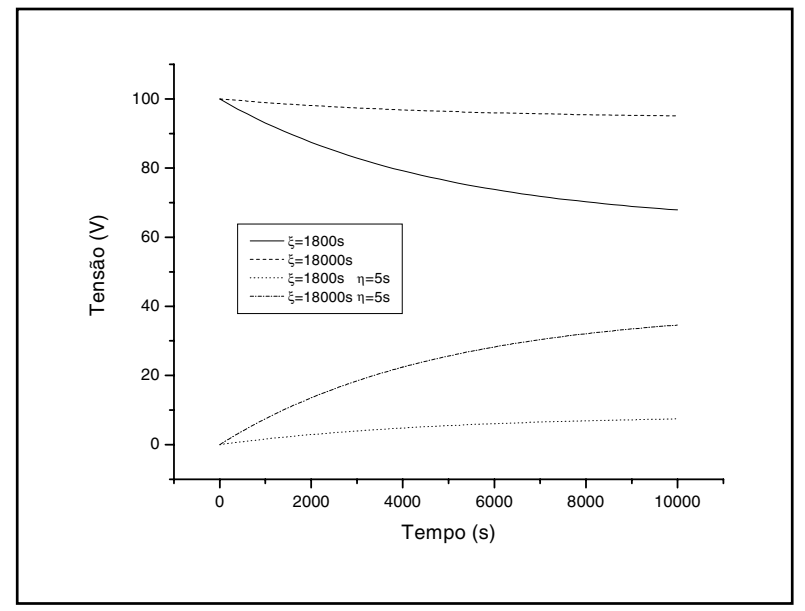

Figura 8. Tensão de retorno e descarga interna em um capacitor de polietileno na hipótese de sua resistência elétrica ser $R=\infty$ com tempo de polarização $\xi$ finitos.

\section{Conclusão}

Embora a grande maioria dos materiais não possua função relaxação dielétrica de forma exponencial, os resultados que se obtém são qualitativamente compatíveis com os experimentos. Seu interesse reside especialmente na simplicidade matemática envolvida. A Eq. (4) permite obter, quando do uso de tensão alternada senoidal de freqüência $\omega$, a permissividade relativa complexa de Debye[10], sendo $1 / \mu$ o tempo de relaxação. Do ponto de vista didático este formalismo é interessante pois permite obter a resposta de um dielétrico nos domínios do tempo e freqüência sem o uso de transformada de Fourier, além de permitir a simulação desta resposta.

É interessante citar que até a Eq. (8) a solução é geral podendo ser aplicada a outras formas da função relaxação dielétrica mais realistas tais como aquelas propostas por Curie-Schweidler, Jonscher, Dissado-Hill etc. O mesmo formalismo pode ser usado em outras áreas, como hereditariedade mecânica (viscoelasticidade) e materiais magnéticos[2].

\section{References}

[1] J. Hopkinson, "Residual Discharge of Layden Jar", Phil. Trans., London, 167, 599 (1877).

[2] J. L. Snoek and F. K. du Fay, Several after-effects Phenomena and residual Losses in Alternating Fields, Philips Technical Review vol.8, n.2, Feb. (1946).

[3] V. Volterra, Theory of Functionals, Dover Pub. Inc. N.Y., 1959. 
[4] B. Gross, "On the Theory of Dielectric Loss," Physical Review, 59, 748 (1941).

[5] B. Gross, Über die Anomalien der Fasten Dielektrika, Zeitschrift Für Physik 107, 217 (1937). B. Gross e P. Rocha, Estudos sobre Dielétricos, An. Acad. Bras. Cien., tomo IX, n.3, 187, (1937). B. Gross e P. Rocha, Estudos sobre Dielectricos II, An. Acad. Bras. Cien., tomo IX, n.4, 309, (1937).

[6] L.A. Dissado and R.M. Hill, "Non Exponencial Decay in Dielectrics and Dynamics of Correlated Systems", Nature, 279, 685 (1979).

[7] D.E. Tilley, A Phenomenological Theory of Dielectric Response, J. of Appl. Physics, 38, 2543 (1967).

[8] F.M. de Oliveira Castro, "On The Integro-Differential Equation of an Absorptive Capacitors", An. Acad. Bras. Cien. 57, 275 (1985).

[9] B. Gross, "On The After-Effects in Solid Dielectrics", Physical Review, 57, 57 (1940).

[10] H. Fröhlich, Theory of Dielectrics, Oxford University Press, Oxford, (1990), p.73.

\section{Apêndice}

\section{Relação entre estímulo e resposta}

Considere um dielétrico onde se aplica no instante $t-\lambda_{k}$ um estímulo em forma de pulso de campo elétrico $\vec{E}\left(t-\lambda_{k}\right)$. Seja $\Delta \vec{P}(t)$ a resposta em polarização medida no instante $t$ conforme mostra a Fig. 9. Supondo existir linearidade entre a resposta e o estimulo podemos escrever

$$
\Delta \vec{P}(t)=\epsilon_{0} \Delta \chi\left(\lambda_{k}\right) \vec{E}\left(t-\lambda_{k}\right)
$$

em que $\epsilon_{0}$ é a permissividade do vácuo e $\Delta \chi\left(\lambda_{k}\right)$ um escalar que representa como a polarização decai para zero no tempo, após a aplicação do pulso de campo elétrico.

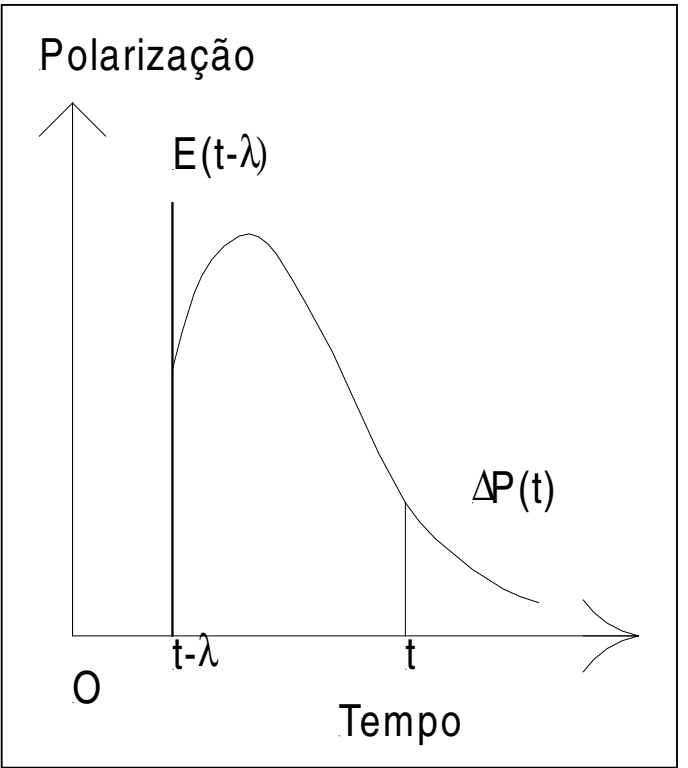

Figura 9. Resposta do dielétrico no instante $t$ devido ao pulso de campo elétrico no instante $t-\lambda$.

Aplicando agora uma seqüência finita de pulsos e admitindo a validez do princípio de superposição podemos escrever

$$
\vec{P}(t)=\sum_{k=1}^{N} \epsilon_{0} \Delta \chi\left(\lambda_{k}\right) \vec{E}\left(t-\lambda_{k}\right),
$$

a qual para um sistema continuo de pulsos se transforma em

$$
\vec{P}(t)=\epsilon_{0} \int_{0^{-}}^{\infty} \chi_{s}(\lambda) \vec{E}(t-\lambda) d \lambda .
$$

A fixação dos extremos da integral (III) esta relacionada ao princípio de causalidade sendo $\chi_{s}(\lambda)=0$ quando $\lambda<0$. Uma propriedade adicional deve ser feita, $\chi_{s}(\lambda) \rightarrow 0$ quando $\lambda \rightarrow \infty$. 